\title{
CALCINEURIN INHIBITOR-INDUCED PAIN SYNDROME IN RENAL TRANSPLANT PATIENT
} Ítalo Magalhães Gusmão1, , Eduardo José do Rosário e Souza ${ }^{1}$, Gustavo Lamego de Barros Costa $^{1}$, Mariana Peixoto Guimarães $_{\text {Ubirajara e Silva de Souza }}$, Marcelo Rocha Scaramussa ${ }^{1}$, Carolina Laís Ladeira Estefani $^{1}$

1.Santa Casa de Belo Horizonte, Belo Horizonte (MG), Brazil.

*Corresponding author: italogusmao23@gmail.com

\section{BACKGROUND}

Calcineurin inhibitor-induced pain syndrome (CIPS) is a rare reaction, probably secondary to bone vasoconstriction induced by these medications. Its most common presentation is a form of symmetrical arthralgia of the knees, ankles and feet. Pain can be severe and disabling, impairing the quality of life of these patients. Diagnosis is made based on the clinical history related to the use of medication and the aid of imaging tests such as magnetic resonance (MRI) and bone scintigraphy.

\section{CASE REPORT}

Male patient, 41 years old, previously with systemic arterial hypertension, hypothyroidism and chronic kidney disease secondary to IgA nephropathy, having undergone kidney transplantation in August 2019, using tacrolimus (TAC), sirolimus and prednisone $5 \mathrm{mg} /$ day, presenting arthralgia in both knees started in January 2021, with mechanical rhythm, without associated morning stiffness. He sought an orthopedist in his city after 15 days of onset of symptoms, being referred to physiotherapy, in which he performed 10 sessions. Initially, the symptoms improved, but it recurred with pain, with the same characteristics. He came to Santa Casa de Belo Horizonte (SCBH) 3 months after the onset of the condition due to the persistence of the symptom. He denied morning stiffness, arthritis, low back pain, xerostomia, xerophthalmia, alopecia, oral ulcers, dysuria, hematuria, dyspnea, and cough. He had an isolated febrile episode $\left(38^{\circ} \mathrm{C}\right)$ on the day of admission. He reported loss of $7 \mathrm{~kg}$ since the onset of joint pain. CAT levels were at the lower limit of normality. Magnetic resonance imaging was performed on the left knee, which showed diffuse signal changes in the distal femoral metaepiphyseal bone marrow and proximal tibiofibular bone marrow associated with distal femoral periosteal reaction. This finding was attributed to CIPS. The patient was kept under observation, with no change in the therapeutic regimen. He returned to the SCBH Nephrology outpatient clinic after one month whose examination showed a reduced serum CAT level, without arthralgia.

\section{CONCLUSION}

Although rare and little recognized, CIPS is a complication that can be found in patients undergoing solid organ or marrow transplantation and using tacrolimus or cyclosporine. Pain can be severe and limiting and its early recognition can help improve the quality of life of these patients.

\section{KEYWORDS}

Kidney transplantation, Arthritis, Calcineurin inhibitor. 